\section{Ocorrência de quedas em idosos residentes em instituições de longa permanência em Pelotas, Rio Grande do Sul, Brasil}

\author{
Falls by elderly people living in long-term care \\ institutions in Pelotas, Rio Grande do Sul State, \\ Brazil
}

\author{
${ }^{1}$ Escola de Saúde, \\ Universidade Católica de \\ Pelotas, Pelotas, Brasil. \\ Correspondência \\ L. M. Álvares \\ Escola de Saúde, \\ Universidade Católica de \\ Pelotas. \\ Rua Rafael Pinto Bandeira \\ 1242, Pelotas, $R S$ \\ 96080-150, Brasil. \\ liegealvares@hotmail.com
}

\begin{abstract}
This study focuses on the occurrence of falls and related factors among elderly individuals living in long-term care institutions in Pelotas, Rio Grande do Sul State, Brazil. A structured questionnaire on demographic characteristics, morbidity, use of psychoactive drugs, and occurrence of falls was used. In December 2006, all 21 long-term care institutions for the elderly registered in the city were contacted, and 19 agreed to participate. A total of 377 elderly were identified, but 96 were unable to answer the questionnaire and 38 refused to answer. Most of the 243 elderly in the sample were women (72.8\%), and mean age was 77.7 years ( $S D=$ 8.9). Nearly one-third of the sample (32.5\%) had suffered at least one fall in the previous year. Falls were twice as frequent in women, individuals with rheumatism or back problems, and those on psychoactive medication. The aim is for the findings to encourage further discussion on the health of elderly residents in long-term care institutions.
\end{abstract}

Accidental Falls; Homes for the Aged; Aged
Liege Mata Álvares 1 Rosângela da Costa Lima 1 Ricardo Azevedo da Silva 1

\section{Introdução}

A Organização Mundial da Saúde (OMS) ${ }^{1}$ considera idoso o indivíduo a partir dos 65 anos em países desenvolvidos e 60 anos, nos países em desenvolvimento 1 . No Brasil, a Política Nacional do Idoso (Lei $\mathrm{n}^{\circ}$. 8.842, Art. $2^{\circ}$ ) entende como idosa a pessoa maior de 60 anos de idade 2 . Atualmente, aproximadamente dois terços da população mundial são idosos e estima-se que poderá chegar a $75 \%$ em 20251 . No Brasil, os idosos representam uma proporção de 8,5\% da população, conforme o Censo Demográfico de 2000 (http://www.ibge.gov.br) e estima-se que em 2020 os idosos poderão chegar a 30 milhões (13\% do total). Dentro deste contexto, com o constante crescimento da população de idosos, vê-se a necessidade de adequado conhecimento sobre os problemas que os atingem ${ }^{3}$. Queda é um dos principais problemas na população idosa 4 . Conforme um consenso desenvolvido pela Associação Médica Brasileira e o Conselho Federal de Medicina, queda é o deslocamento não intencional do corpo para um nível inferior à posição inicial com incapacidade de correção de tempo hábil, determinado por circunstâncias multifatoriais, comprometendo a estabilidade 5 . Perto de um terço da população idosa residente na comunidade sofre quedas e metade dela experiencia múltiplas quedas a cada ano. Os idosos residentes em instituições de longa permanência para idosos apresentam três vezes mais chances 
de cair do que os que residem na comunidade (Rubenstein et al., 1994, apud Vu et al. 4).

No entanto, a institucionalização dos idosos constitui, muitas vezes, a única opção para esses indivíduos e suas famílias ${ }^{6}$. Mesmo que haja um regulamento técnico definindo normas para instituições de longa permanência para idosos 2 , ainda assim muitas funcionam sem as condições ideais 6,7. Além disto, os idosos, ao serem transferidos do próprio lar para uma instituição, se deparam com uma transformação do seu estilo de vida, sendo desviados de todo seu projeto existencial 8 .

Estudos internacionais realizados com idosos em instituições de longa permanência apresentaram diferentes freqüências de quedas como nos Estados Unidos 42,9\% (34.163 idosos em 437 instituições) 9 e 49\% (462 idosos em 56 instituições) 10, no Canadá 10,6\% (14.744 idosos em 71 instituições) 11, na Austrália 11\% (2.005 idosos em 81 instituições) 12 no Japão 12,5\% (746 idosos em 10 instituições) 13 , no Chile 24\% (453 idosos em 8 instituições) 14, na Colômbia 36\% (116 idosos em uma instituição) 15 e em Cuba 78\% (23 idosos em uma instituição) ${ }^{16}$. Do mesmo modo, os estudos brasileiros apresentam $40 \%$ (105 idosos em 4 instituições) em São Paulo 17, 64,6\% (312 idosos em um asilo) na Bahia 18 , e o Instituto Nacional de Traumato-Ortopedia (INTO) 19 faz uma estimativa de $50 \%$ de quedas de idosos residentes em instituições asilares brasileiras.

Há muitos estudos que mostram os fatores de risco relacionados à queda 4,9,13,16,17,18,20,21,22,23, $24,25,26,27,28,29$, mas eles podem diferir conforme a cidade ou país, dependendo das diferenças culturais e das condições de vida desses idosos 30 . As conseqüências não são somente físicas 5,11, $16,21,23,24,25,28,29,31,32$, mas psicológicas 33,34 e sociais como hospitalização 21,23,24,29,34, institucionalização 24,31 ou reabilitação 24,34 , diminuindo autonomia e aumentando custos para a saúde pública $3,24,31,35$.

Neste contexto, o objetivo do presente estudo foi descrever a ocorrência de quedas e fatores associados entre idosos residentes em instituições de longa permanência na cidade de Pelotas, Rio Grande do Sul, Brasil.

\section{Métodos}

Foi realizado um estudo transversal com idosos residentes em todas as instituições de longa permanência para idosos registradas na Prefeitura de Pelotas, até dezembro de 2006. As instituições foram caracterizadas por letras com a finalidade de garantir o sigilo.
Os idosos residentes nestas instituições que assinaram o termo de consentimento foram entrevistados. É considerado idoso, em países em desenvolvimento, aquele com idade a partir de 60 anos 1,2. Foram excluídos da amostra os indivíduos com menos de 60 anos e também aqueles que não tinham condições de responder o questionário. A coleta de dados realizou-se nas próprias instituições, no mês de dezembro de 2006, por meio de um questionário padronizado e pré-codificado. Foram obtidas informações sobre: idade (em anos completos); sexo (feminino e masculino); presença de morbidade referida (doença de coluna ou costas, artrite ou reumatismo, diabetes ou açúcar no sangue, bronquite ou asma, pressão alta, doença do coração). Tais morbidades foram incluídas por serem mais freqüentes em idosos e que podem levar a alterações posturais, desconforto e dor, podendo causar quedas. Também foi avaliado o estado cognitivo pelo teste MiniExame do Estado Mental (MEEM), com aplicação da versão brasileira conforme Bertolucci et al. 36 . Os dados sobre quedas incluíram perguntas sobre número de quedas no último ano; parte do corpo atingida (membros inferiores, cabeça, tronco, membros superiores, quadril, nenhuma); conseqüências da queda (nenhuma, fratura, equimose, entorse, edema, ou outros); local da ocorrência (rua, quarto, banheiro, pátio e outros locais); turno (manhã, tarde, noite); motivo (escorregão, tropeção, desequilíbrio, tontura, ou outro motivo). Caso fosse relatada mais do que uma queda, eram registradas informações sobre a última. A utilização de medicamentos psicotrópicos (número e tipo de medicamento) foi colhida do prontuário do idoso, visto que alguns idosos não sabiam qual o nome do medicamento que utilizavam. Para realização das entrevistas colaborou um grupo de acadêmicos voluntários, da Universidade Católica de Pelotas, previamente treinados.

Para a formação do banco de dados do estudo foi utilizado o programa Epi Info versão 6.04 (Centers for Disease Control and Prevention, Atlanta, Estados Unidos). Foi realizada dupla digitação dos questionários, já revisados e codificados, checagem automática de amplitude e análise de inconsistências para evitar possíveis erros.

$\mathrm{Na}$ análise dos dados, foram utilizados os programas SPSS versão 13.0 (SPSS Inc., Chicago, Estados Unidos) e Stata versão 10.0 (Stata Corp., College Station. Estados Unidos). Inicialmente, foi realizada uma análise descritiva para conhecer a prevalência do desfecho e dos possíveis fatores de risco e análise bivariada para testar a associação de quedas com as variáveis independentes utilizando o teste exato de Fisher. Posteriormente, as razões de prevalência foram 
calculadas por regressão multivariada (regressão de Poisson) de acordo com modelo teórico elaborado pelos autores. No primeiro nível, foram incluídas as variáveis idade e sexo; no segundo, a morbidade referida pelos idosos e, no último nível, o uso de psicotrópicos. Permaneceram no modelo todas as variáveis com $\mathrm{p}<0,20$.

O projeto foi aprovado pelo Comitê de Ética da Universidade Católica de Pelotas.

\section{Resultados}

Em Pelotas, no mês de dezembro de 2006, foram visitadas todas as 21 instituições de longa permanência para idosos registradas na cidade. Destas, 19 consentiram em participar do estudo e duas instituições (uma com 11 e outra com 10 idosos) não permitiram a realização das entrevistas. Foram identificados 377 idosos nestas instituições. Dentre eles, 38 recusaram-se a responder e 96 não tinham condições de compreensão das perguntas. Assim, 243 idosos (73,4\%) responderam questões sobre quedas e demais variáveis independentes.

A idade média deste grupo foi de 77,7 anos (desvio-padrão- $\mathrm{DP}=8,9$ ), sendo a idade média das mulheres 79,3 anos ( $\mathrm{DP}=8,3$ ) e dos homens 73,5 anos (DP = 9,1). As demais características gerais da população estudada são apresentadas na Tabela 1. As mulheres representaram a maioria da amostra com 72,8\% ( $=177)$. Dentre as morbidades investigadas, as mais referidas foram doença da coluna (40\%), hipertensão $(37,2 \%)$ e artrite ou reumatismo $(32,1 \%)$. Foram encontrados $59,7 \%$ dos idosos usando medicação psicotrópica e 55,6\% dos idosos com déficit cognitivo. Cerca de um terço dos idosos $(32,5 \%)$ sofreu pelo menos uma queda no último ano. Um terço dos idosos apresentou uma queda, duas quedas foram referidas por $22,5 \%$ e três ou mais quedas por $35,2 \%$. A ocorrência de quedas variou de $16,7 \%$ na instituição I e $62,5 \%$ na instituição O.

Na Tabela 2, encontram-se as descrições das quedas. As partes do corpo mais atingidas foram os membros inferiores (32\%), cabeça $(26,7 \%)$ e tronco (16\%); as principais conseqüências foram equimose $(25,4 \%)$, nenhuma $(22,2 \%)$, fratura e outros $(20,6 \%)$, entorse $(6,3 \%)$ e edema $(4,8 \%)$. Os locais onde mais ocorreram quedas foram: a rua (30,9\%), o quarto (25\%), o banheiro $(17,6 \%)$, o pátio e outros locais $(13,2 \%)$, e o turno de maior ocorrência foi o diurno (85,8\%). A ocorrência de quedas ocorreu nos turnos tarde (50,7\%), manhã $(35,2 \%)$ e noite $(14,1 \%)$. Dentre os motivos da queda citados, o maior percentual foi escorregão $(23,6 \%)$, seguido de tontura $(22,2 \%)$, desequilí- brio (16,7\%), tropeção (12,5\%) e outros motivos (25\%).

A Tabela 3 apresenta a ocorrência das quedas conforme as variáveis independentes, a análise bruta e ajustada. A ocorrência de quedas foi de $37,3 \%$ no sexo feminino e $19,7 \%$ no masculino $(\mathrm{p}=0,009)$. Os idosos entre 60 e 69 anos de idade tiveram $37,8 \%$ de quedas, entre 70 e 79 anos tiveram $34,5 \%$ de quedas e aqueles com 80 ou mais anos tiveram $30 \%$ de quedas, mostrando que o aumento da faixa etária foi inversamente proporcional ao relato de quedas na população estudada, embora essas diferenças não tenham sido estatisticamente significativas $(p=0,6)$. Os idosos com reumatismo e doença da coluna apresentaram maior prevalência de quedas no último ano com $48 \%$ ( $p=0,001)$ e $42 \%(p=0,01)$, respectivamente. A presença das demais doenças investigadas - diabetes, hipertensão, bronquite/asma e déficit cognitivo - não apresentou associação estatisticamente significativa com a ocorrência de quedas. Os idosos que faziam uso de psicotrópicos tiveram $40 \%$ de quedas e os que não utilizavam tal tipo de medicação tiveram $21,4 \%$, evidenciando que a utilização de medicação psi-

Tabela 1

Características dos idosos residentes em instituições de longa permanência para idosos. Pelotas, Rio Grande do Sul, Brasil, 2006 ( $N=243)$.

\begin{tabular}{|c|c|c|}
\hline Variável & $\mathrm{n}$ & $\%$ \\
\hline \multicolumn{3}{|l|}{ Idade (anos) } \\
\hline $60-69$ & 45 & 18,8 \\
\hline $70-79$ & 84 & 35,1 \\
\hline 80 ou mais & 110 & 46,0 \\
\hline \multicolumn{3}{|l|}{ Sexo } \\
\hline Feminino & 177 & 72,8 \\
\hline Masculino & 66 & 27,2 \\
\hline \multicolumn{3}{|l|}{ Morbidade referida } \\
\hline Doença da coluna/costas & 96 & 40,0 \\
\hline Artrite/Reumatismo & 75 & 32,1 \\
\hline Hipertensão arterial sistêmica & 86 & 37,2 \\
\hline Cardiopatias & 58 & 25,3 \\
\hline Diabetes & 35 & 14,9 \\
\hline Bronquite/Asma & 25 & 10,5 \\
\hline \multicolumn{3}{|l|}{ Déficit cognitivo } \\
\hline Sim & 129 & 55,6 \\
\hline Não & 103 & 44,4 \\
\hline \multicolumn{3}{|l|}{ Uso de psicotrópicos } \\
\hline Sim & 145 & 59,7 \\
\hline Não & 98 & 40,3 \\
\hline
\end{tabular}


Tabela 2

Descrição das quedas ocorridas entre idosos residentes nas instituições de longa permanência para idosos. Pelotas, Rio Grande do Sul, Brasil, 2006 (N = 79).

\begin{tabular}{|c|c|c|}
\hline Variável & $\mathbf{n}$ & $\%$ \\
\hline \multicolumn{3}{|l|}{ Partes do corpo atingidas } \\
\hline Membros inferiores & 24 & 32,0 \\
\hline Cabeça & 20 & 26,7 \\
\hline Tronco & 12 & 16,0 \\
\hline Membros superiores & 9 & 12,0 \\
\hline Quadril & 6 & 8,0 \\
\hline Nenhuma & 4 & 5,3 \\
\hline \multicolumn{3}{|l|}{ Conseqüências da queda } \\
\hline Equimose & 16 & 25,4 \\
\hline Nenhuma & 14 & 22,2 \\
\hline Fratura & 13 & 20,6 \\
\hline Outros & 13 & 20,6 \\
\hline Entorse & 4 & 6,3 \\
\hline Edema & 3 & 4,8 \\
\hline \multicolumn{3}{|l|}{ Local de ocorrência } \\
\hline Rua & 21 & 30,9 \\
\hline Quarto & 17 & 25,0 \\
\hline Banheiro & 12 & 17,6 \\
\hline Pátio & 9 & 13,2 \\
\hline Outros locais & 9 & 13,2 \\
\hline \multicolumn{3}{|l|}{ Turno de ocorrência } \\
\hline Tarde & 36 & 50,7 \\
\hline Manhã & 25 & 35,2 \\
\hline Noite & 10 & 14,1 \\
\hline \multicolumn{3}{|l|}{ Motivo da queda } \\
\hline Outros & 18 & 25,0 \\
\hline Escorregão & 17 & 23,6 \\
\hline Tontura & 16 & 22,2 \\
\hline Desequilíbrio & 12 & 16,7 \\
\hline Tropeção & 9 & 12,5 \\
\hline \multicolumn{3}{|l|}{ Número de quedas } \\
\hline Uma & 30 & 42,3 \\
\hline Três ou mais & 25 & 35,2 \\
\hline Duas & 16 & 22,5 \\
\hline
\end{tabular}

cotrópica esteve associada à maior prevalência de acidentes por quedas $(\mathrm{p}=0,003)$. Após a análise multivariada, as mulheres apresentaram 2,18 vezes (intervalo de 95\% de confiança - IC95\%: $1,18 ; 4,04)$ mais quedas que os homens. Os idosos que tomaram medicamentos psicotrópicos tiveram 1,68 vezes (IC95\%: 1,01; 2,79) mais quedas do que aqueles que não utilizaram.

\section{Discussão}

Neste estudo foi encontrada uma ocorrência de $32,5 \%$ de quedas em idosos residentes em instituições de longa permanência. Este percentual é inferior ao reportado em dois estudos nacionais, cuja freqüência variou de $40 \%$ a $64,6 \% 17,18$. O estudo transversal de Lojudice 17 entrevistou 105 idosos de quatro instituições em Catanduva, São Paulo, encontrando uma ocorrência de $40 \%$. Já o estudo de Santos 18, ao final de oito meses de acompanhamento, encontrou uma freqüência de $64,6 \%$ de quedas em 312 idosos de uma instituição de Salvador, Bahia. A maior prevalência de quedas encontrada neste último estudo pode ser devido ao delineamento da pesquisa ou porque obteve as informações sobre as quedas mediante dois instrumentos, a saber, entrevista com o próprio idoso e consulta ao prontuário preenchido pelos funcionários das instituições. Vale ressaltar que os funcionários foram treinados para anotar no prontuário dos idosos todas as quedas ocorridas. Na literatura internacional, esta freqüência variou de $10,6 \%$ a $49 \%$ de quedas 10,12,13,14,37,38, exceto um estudo realizado em Cuba, com apenas 23 idosos de uma instituição, que encontrou uma freqüência de quedas de $78 \% 16$.

O turno de maior ocorrência de quedas foi o diurno com $86 \%$ e esse dado também é um achado na literatura $15,18,20,21,39$. O fato contraria a idéia de que o idoso tem mais risco de cair quando levanta durante a noite para ir ao banheiro ou para beber água 20 .

O presente estudo evidenciou uma ocorrência maior de quedas na rua $(30,9 \%)$, no quarto (25\%) e no banheiro (17,6\%). Há uma possibilidade de o idoso estar mais desprotegido quando sai na rua. Lojudice 17 encontrou como os lugares onde os idosos mais caíram o banheiro $(33,3 \%)$, seguido do quarto e da sala com mesmo percentual (16,7\%). Estudos demonstram que idosos tendem a sofrer mais quedas em seus lares 5,20,31,39 e nos cômodos mais utilizados 18 . O menor número de quedas fora destes ambientes pode ocorrer em razão da menor socialização dos idosos 3, especialmente daqueles institucionalizados 6,8 , o que contradiz a realidade do presente estudo.

Santos 18 aponta a transferência de posição, como de estar sentado ou deitado para ficar em pé ou o inverso, como importante fator de risco para a queda. Dentre os motivos para a ocorrência de quedas, o estudo encontrou com maior freqüência: escorregão (23,6\%), sentir tontura $(22,2 \%)$ e desequilíbrio (16,7\%). De maneira semelhante, o estudo de Rubenstein 23 cita, como causa acidental, desequilibrar (17\%) e vertigem (13\%) e Lojudice 17 também apontou falta de 
Razão de prevalências (RP) de quedas de idosos residentes em instituições de longa permanência para idosos conforme as variáveis independentes. Pelotas, Rio Grande do Sul, Brasil, 2006 ( $N=243$ ).

\begin{tabular}{|c|c|c|c|c|}
\hline Variável & $\mathbf{n}$ & $\%$ & RP (IC95\%) & $\begin{array}{l}\text { RP ajustada } \\
\text { (IC95\%) }\end{array}$ \\
\hline Idade (anos) & & $p=0,6$ & $p=0,71$ & $p=030$ \\
\hline $60-69$ & 45 & 37,8 & 1,00 & 1,00 \\
\hline $70-79$ & 84 & 34,5 & $0,91(0,50 ; 1,66)$ & $0,76(0,41 ; 1,41)$ \\
\hline 80 e mais & 110 & 30,0 & $0,79(0,44 ; 1,42)$ & $0,62(0,34 ; 1,14)$ \\
\hline Sexo & & $p=0,009$ & $p=0,04$ & $p=0,01$ \\
\hline Masculino & 66 & 19,7 & 1,0 & 1,0 \\
\hline Feminino & 177 & 37,3 & $1,89(1,04 ; 3,43)$ & $2,18(1,18 ; 4,04)$ \\
\hline Artrite/Reumatismo & & $p=0,001$ & $p=0,007$ & $p=0,13$ \\
\hline Não & 159 & 25,8 & 1,00 & 1,00 \\
\hline Sim & 75 & 48,0 & $1,86(1,19 ; 2,91)$ & $1,52(0,89 ; 2,61)$ \\
\hline Doenças da coluna & & $p=0,01$ & $p=0,03$ & $p=0,08$ \\
\hline Não & 144 & 26,4 & 1,00 & 1,00 \\
\hline Sim & 96 & 42,7 & $1,61(1,04 ; 2,51)$ & $1,68(0,94 ; 3,01)$ \\
\hline Cardiopatia & & $p=0,1$ & $p=0,19$ & $p=0,25$ \\
\hline Não & 171 & 29,8 & 1,00 & 1,00 \\
\hline Sim & 58 & 41,4 & $1,39(0,85 ; 2,25)$ & $1,42(0,78 ; 2,56)$ \\
\hline Diabetes & & $p=0,1$ & $p=0,22$ & $p=0,74$ \\
\hline Não & 200 & 30,0 & 1,00 & 1,00 \\
\hline Sim & 35 & 42,9 & $1,43(0,81 ; 2,52)$ & $0,89(0,46 ; 1,75)$ \\
\hline Hipertensão & & $p=0,6$ & $p=0,69$ & $p=0,47$ \\
\hline Não & 145 & 31,7 & 1,00 & 1,00 \\
\hline Sim & 86 & 34,9 & $1,10(0,69 ; 1,74)$ & $0,81(0,46 ; 1,43)$ \\
\hline Bronquite/Asma & & $p=0,8$ & $p=0,79$ & $p=0,88$ \\
\hline Não & 214 & 32,7 & 1,00 & 1,00 \\
\hline $\operatorname{Sim}$ & 25 & 36,0 & $1,10(0,55 ; 2,20)$ & $1,06(0,51 ; 2,18)$ \\
\hline Déficit cognitivo & & $p=0,1$ & $p=0,17$ & $p=0,10$ \\
\hline Não & 103 & 26,2 & 1,00 & 1,00 \\
\hline Sim & 129 & 36,4 & $1,39(0,87 ; 2,23)$ & $1,55(0,92 ; 2,62)$ \\
\hline $\begin{array}{l}\text { Uso de psicotrópicos } \\
\text { (último mês) }\end{array}$ & & $p=0,003$ & $p=0,01$ & $p=0,05$ * \\
\hline Não & 98 & 21,4 & 1,00 & 1,00 \\
\hline Sim & 145 & 40,0 & $1,87(1,13 ; 3,08)$ & $1,68(1,01 ; 2,79)$ \\
\hline
\end{tabular}

IC95\%: intervalo de 95\% de confiança.

* Ajustado para idade, sexo e reumatismo.

equilíbrio (24\%) e tontura (11,9\%) como causas. Depois que o idoso se desequilibra, geralmente não apresenta mais condições de voltar à posição inicial, podendo necessitar, neste momento, de um ponto de apoio para auxílio como bengala, ou corrimão ${ }^{23}$. No estudo de Coutinho et al. 39, caso controle realizado em cinco hospitais no Rio de Janeiro, para investigar fatores de risco para fraturas graves decorrentes de quedas em idosos, os pisos escorregadios foram atribuídos como a causa para quedas.
Com relação à parte do corpo atingida, as mais atingidas foram membros inferiores (32\%), cabeça $(26,7 \%)$, tronco (16\%), membros superiores (12\%) e quadril (8\%). Tais valores estão de acordo com os da literatura, que aponta como parte do corpo mais atingida a região proximal do fêmur 4,31,39 ou quadril ${ }^{9}$, sendo nessa mesma região do corpo encontrada a conseqüência de maior impacto: a fratura 4,21,28,31,37,39. No entanto, depois que a queda ocorre, é difícil identificar se houve fratura e por isso o idoso caiu ou se a fra- 
tura foi conseqüência da queda. Para Lebrão 40, possivelmente, essa maior freqüência de queda entre mulheres seria um indicativo de que nesse sexo pode ocorrer mais a fratura anterior à queda, já que em 22,3\% delas o médico constatou osteoporose, fato que ocorreu apenas com 2,7\% dos homens. Os idosos estudados nesta pesquisa apresentaram uma freqüência de $19,7 \%$ de fratura, além desta conseqüência, foram agrupadas outras, como entorse, edema e equimose com $39,4 \%$ e demais conseqüências com 19,7\%. A revisão de Rubenstein 23 aponta que $10 \%$ a $25 \%$ das quedas resultam em fratura ou laceração. Outros estudos 14,16,41 também mostram os demais traumas como conseqüências decorrentes de quedas.

A história prévia de quedas tem relação com a recorrência de quedas 39,42 . A possibilidade de o idoso ter experimentado uma queda anterior com conseqüência séria, como fratura, parece imputar uma maior vulnerabilidade a novos episódios 43 . No presente estudo, dos 32,5\% idosos que sofreram quedas, $42,3 \%$ tiveram apenas uma queda, 22,5\% duas quedas e 35,2\% tiveram três ou mais quedas. No estudo de Perracini \& Ramos 43 , $30,9 \%$ dos idosos entrevistados relataram ter caído no ano anterior ao primeiro inquérito e, dentre eles, $10,8 \%$ relataram duas ou mais quedas, no segundo inquérito, 29,1\% dos idosos referiram ter caído no ano anterior e 12,4\% caíram de forma recorrente.

A média de idade (77,7 anos) no presente estudo foi semelhante aos estudos de Chaimowicz \& Greco 44 (77,0 anos) em 33 instituições com 1.128 idosos e de Romero 15 (78,0 anos) em uma instituição com 116 idosos. A literatura mostra que quanto maior a idade do idoso, maior é o risco de queda visto que a idade avançada está intimamente ligada a outros fatores de exposição a quedas 5,16,18,21. Contudo, no presente estudo não houve esta relação, pelo contrário, 37,8\% dos idosos que tinham entre 60 e 69 anos caíram, $34,5 \%$ dos idosos entre 70 e 79 anos caíram e $30 \%$ dos idosos com 80 anos ou mais caíram. Isso revela que a idade foi um fator protetor para quedas. Romero 15 também encontrou no seu estudo de coorte em uma instituição com 116 idosos, o aumento da idade como proteção para a queda. A razão para esse fato pode ser a menor deambulação dos idosos com maior idade. Durante o dia, provavelmente, por não possuírem força suficiente nos membros inferiores para deambular, pois conforme Matsudo \& Barros Neto 45 a força muscular declina com a idade e, sobretudo, com a idade mais avançada, uma vez que há uma perda gradual de $10 \%$ por década a partir dos 50 anos. À medida que aumenta a idade cronológica, diminui a atividade dos idosos 46 junto a isso pode vir uma proteção para quedas, já que ficam menos expostos aos obstáculos ambientais. Outra explicação poderia ser que os idosos poderiam não recordar suas quedas.

Assim como em outros estudos 14,15,20,31,40, $42,43,44$, o sexo feminino representa a maior parte da população de idosos, pois as mulheres idosas vivem mais do que os homens 46 . No presente estudo, a população feminina foi de $72,8 \%$. Além disso, as mulheres sofreram cerca de duas vezes mais quedas que os homens. Na literatura também foi observada uma maior ocorrência de quedas entre as mulheres 5,14,18,40,43,47. Para Gac et al. 14 as quedas ocorrem mais no sexo feminino devido ao menor estado funcional, maior morbidade e maior presença de osteoartrose. As mulheres também apresentam maior perda de massa óssea por causa da redução do estrógeno a partir dos 40 anos de idade, contribuindo para deteriorar o seu estado funcional 40 . Segundo Perracini \& Ramos 43, a maior chance de queda ocorre nas mulheres idosas por conta da maior fragilidade em relação aos homens, maior prevalência de doenças crônicas, assim como maior vínculo às atividades domésticas. Fried et al. 47 também relataram que o sexo feminino tem maior probabilidade de se tornar mais frágil que o sexo masculino pela menor qualidade e força da massa muscular.

Alguns estudos 31,48,49 apontam que as doenças articulares são freqüentes na população idosa e que contribuem para redução da capacidade física, ocasionando dor, influenciando no equilíbrio ou controle postural, favorecendo a ocorrência de quedas. Entretanto, no presente estudo não houve relação significativa entre a ocorrência de quedas e reumatismo e patologias decorrentes da coluna com as quedas.

As demais patologias estudadas como asma, diabete, cardiopatia, hipertensão arterial e déficit cognitivo não tiveram associação significativa com ocorrência de quedas. Isso pode ter ocorrido em razão de o tamanho da amostra ser pequena, ou porque os idosos poderiam não recordar suas enfermidades, enquanto em alguns estudos 10,12,18,24,26,38 a relação entre queda e as comorbidades podem ter ocorrido devido às informações serem colhidas de maneira mais minuciosa, dado que os autores permaneceram de 23 a 365 dias colhendo dados; e em outros estudos 24,37 , os dados foram verificados de 1.460 a 1.825 dias. Romero 15 também não encontrou resultados estatisticamente significativos entre enfermidades cardiovasculares e hipertensão arterial quando relacionadas com quedas; para Lojudice 17 não houve evidência de associação entre queda e presença de derrame, labirintite, convulsão, Parkinson, asma, diabetes e arritmias, 
existindo associação estatisticamente significativa entre queda e presença de doenças reumáticas $(32 \%$ e $\mathrm{p}<0,001)$. Resultados afins do presente estudo foram encontrados em Lojudice 17 talvez por apresentarem semelhanças como delineamento transversal e o instrumento de coleta de dados. A alteração cognitiva é um fator de risco para queda comumente identificado por causa da desorientação viso-espacial, levando a uma má percepção dos perigos ambientais e a uma avaliação errônea das próprias habilidades $13,18,23,24,25$. Em contraste com esta literatura, este estudo não encontrou uma relação significativa entre queda e o déficit cognitivo. Tal achado está de acordo com a metanálise e revisão sistemática de Oliver et al. 34 , que também não mostrou uma associação significativa entre quedas e fraturas e prevalência de demência ou de estado cognitivo alterado. $\mathrm{O}$ autor relata que este fato é devido aos inúmeros testes utilizados para demência. Também pode ser por conta do estilo de vida mais sedentário dos idosos, pois como estão comprometidos mentalmente, os cuidadores geralmente lhes impõem uma maior limitação funcional, seja em cadeiras ou em suas camas, diminuindo, assim, as chances de queda. Além disso, os idosos sem condições de responder o questionário, geralmente com demência, foram excluídos do estudo.

A utilização de psicotrópicos esteve significativamente associada com quedas no presente estudo. A maioria das referências 14,18,20,21,23, 28,32,37,38,41 aponta que medicação psicotrópica pode levar o idoso a cair por causarem hipotensão postural, sedação excessiva e diminuição no tempo de reação, dificuldades no equilíbrio e no caminhar, arritmias e danos a um estado de alerta cognitivo. Associado a essas drogas existe o risco de severas fraturas 29. Apenas o estudo de Avidan et al. ${ }^{9}$ sugere que o uso de hipnóticos em idosos institucionalizados foi protetor para ocorrência de quedas, uma vez que quem tem insônia pode estar mais predisposto a sofrer queda do que aqueles que usam essa medicação.

O presente estudo apresenta limitações que decerto deveriam ser melhoradas em futuros estudos. Fatores tempo-dependentes como doenças agudas, a introdução de novas medicações e tempo de uso de medicações quando a queda ocorrida não foi bem documentada porque o prontuário não continha semelhantes informações. Estudo de Avidan et al. 9 em Michigan, nos Estados Unidos, propõem um instrumento, Long-Term Care Facility Resident Assessment Instrument, contendo 350 itens sobre a saúde do idoso, que é atualizado no mínimo quatro vezes por ano. Para aplacar essa falta, poder-se-ia utilizar uma entrevista com os funcionários para fornecer informações precisas ou suficientes sobre cada idoso como em outros estudos 18,20,30. Fatores ambientais tais como a presença de corrimão ou chão antiderrapante etc., importantes para proteção dos idosos, não foram avaliados nas instituições. Tal fato ocorreu em razão da dificuldade de acesso às instituições para avaliação do local quanto ao seu adequado funcionamento.

Apesar de todas as dificuldades de entrevistar os idosos, este é um dos primeiros estudos a abordar este problema de quedas em idosos institucionalizados na cidade. Pretende-se que as informações obtidas possibilitem discussões sobre a saúde do idoso que residem em instituições de longa permanência. 


\section{Resumo}

O objetivo deste estudo foi descrever a ocorrência de quedas e fatores associados em idosos residentes em instituições de longa permanência para idosos em Pelotas, Rio Grande do Sul, Brasil. Foi utilizado um questionário estruturado com perguntas sobre características demográficas, morbidade, uso de psicotrópicos e ocorrência de quedas. Em dezembro de 2006, foram visitadas todas as 21 instituições de longa permanência para idosos registradas na cidade. Dentre elas, 19 consentiram em participar do estudo. Foram identificados 377 idosos, mas 96 não tinham condições de responder o questionário e 38 foram recusas. Dos 243 idosos incluídos no estudo, a maioria era mulheres (72,8\%) e tinha, em média, 77,7 (DP = 8,9) anos. Cerca de um terço dos idosos (32,5\%) sofreu pelo menos uma queda no último ano. A ocorrência de quedas foi duas vezes maior nas mulheres, nos idosos com reumatismo ou doença da coluna e naqueles que utilizavam medicação psicotrópica. Pretende-se que as informações obtidas possibilitem discussões sobre a saúde dos idosos residentes em instituições de longa permanência.

Acidentes por Quedas; Instituições de Longa Permanência para Idosos; Idoso

\section{Referências}

1. Organização Mundial da Saúde. Ageing and life course. http://www.who.int/ageing/en (acessado em 01/Jul/2007).

2. Casa Civil, Presidência da República. Lei no ${ }^{\circ} .8 .842$, de 4 de janeiro de 1994. http://www.planalto.gov. br/ccivil_03/LEIS/L8842.htm (acessado em 25/ Set/2009).

3. Chaimowicz F. A saúde dos idosos brasileiros às vésperas do século XXI: problemas, projeções e alternativas. Rev Saúde Pública 1997; 31:184-200.

4. Vu MQ, Weintraub N, Rubenstein LZ. Falls in the nursing home: are they preventable? J Am Med Dir Assoc 2005; 6:82-7.

5. Pereira SRM, Buksman S, Perracini M, Py L, Barreto KML, Leite VMM. Projeto Diretrizes: quedas em idosos. Rio de Janeiro: Sociedade Brasileira de Geriatria e Gerontologia; 2001.

6. Davim RM, Torres GV, Dantas SM, Lima VM. Study with elderly from asylums in Natal/RN: socioeconomic and health characteristics. Rev Latinoam Enferm 2004; 12:518-24.

\section{Colaboradores}

L. M. Álvares e R. C. Lima participaram de todas as etapas da elaboração do artigo. R. A. Silva colaborou na redação do artigo e na aprovação final da versão a ser publicada.
7. Yamamoto A, Diogo MJ. The elderly and asylums at the municipality of Campinas. Rev Latinoam Enferm 2002; 10:660-6.

8. Freire Júnior RC, Tavares MFL. A saúde sob o olhar do idoso institucionalizado: conhecimento e valorizando sua opinião. Interface Comun Saúde Educ 2005; 9:147-58.

9. Avidan AY, Fries BE, James ML, Szafara KL, Wright GT, Chervin RD. Insomnia and hypnotic use, recorded in the minimum data set, as predictors of falls and hip fractures in Michigan nursing homes. J Am Geriatr Soc 2005; 53:955-62.

10. Hill-Westmoreland EE, Gruber-Baldini AL. Falls documentation in nursing homes: agreement between the minimum data set and chart abstractions of medical and nursing documentation. J Am Geriatr Soc 2005; 53:268-73. 
11. Brennan nee Saunders J, Johansen A, Butler J, Stone M, Richmond P, Jones S, et al. Place of residence and risk of fracture in older people: a population-based study of over 65-year-olds in Cardiff. Osteoporos Int 2003; 14:515-9.

12. Hien le TT, Cumming RG, Cameron ID, Chen JS, Lord SR, March LM, et al. Atypical antipsychotic medications and risk of falls in residents of aged care facilities. J Am Geriatr Soc 2005; 53:1290-5.

13. Izumi K, Makimoto K, Kato M, Hiramatsu T. Prospective study of fall risk assessment among institutionalized elderly in Japan. Nurs Health Sci 2002; 4:141-7.

14. Gac H, Marín PP, Castro S, Hoyl T, Valenzuela E. Caídas en adultos mayores institucionalizados: descripción y evaluación geriátrica. Rev Méd Chile 2003; 131:887-94.

15. Romero C, Uribe M. Factores de riesgo para que la población mayor institucionalizada presente caídas. Rev Cienc Salud (Bogotá) 2004; 2:91-110.

16. Díaz Oquendo D, Barrera García AC, Pacheco Infante A. Incidencia de las caídas en el adulto mayor institucionalizado. Rev Cuba Enferm 1999; 15:34-8.

17. Lojudice DC. Quedas de idosos institucionalizados: ocorrência e fatores associados [Dissertação de Mestrado]. Ribeirão Preto: Faculdade de Medicina de Ribeirão Preto, Universidade de São Paulo; 2005.

18. Santos MLC, Andrade MC. Incidência de quedas relacionada aos fatores de riscos em idosos institucionalizados. Rev Baiana Saúde Pública 2005; 29:57-68.

19. Instituto Nacional de Traumatologia e Ortopedia. Dicas dos especialistas: quedas em idosos. http:// www.into.saude.gov.br/de_quedas_idosos.php (acessado em 18/Jul/2007).

20. Carvalho AM, Coutinho ESF. Demência como fator de risco para fraturas graves em idosos. Rev Saúde Pública 2002; 36:448-54.

21. Coutinho ESF, Silva SD. Uso de medicamentos como fator de risco para fratura grave decorrente de queda em idosos. Cad Saúde Pública 2002; 18:1359-66.

22. Lord SR, Menz HB, Sherrington C. Home environment risk factors for falls in older people and the efficacy of home modifications. Age Ageing 2006; 35:55-9.

23. Rubenstein LZ. Falls in older people: epidemiology, risk factors and strategies for prevention. Age Ageing 2006; 35:37-41.

24. Mahoney JE, Glysch RL, Guilfoyle SM, Hale LJ, Katcher ML. Trends, risk factors, and prevention of falls in older adults in Wisconsin. WMJ 2005; 104:22-8.

25. Duque G, Mallet L, Roberts A, Gingrass S, Kremer R, Sainte-Marie LG, et al. To treat or not to treat, that is the question: proceedings of the Quebec symposium for the treatment of osteoporosis in longterm care institutions, Saint-Hyacinthe, Quebec, November 5, 2004. J Am Med Dir Assoc 2007; 8(3 Suppl 2):e67-73.

26. Turcu A, Toubin S, Mourey F, D'Athis P, Manckoundia P, Pfitzenmeyer P. Falls and depression in older people. Gerontology 2004; 50:303-8.
27. Ozcan A, Donat H, Gelecek N, Ozdirenc M, Karadibak D. The relationship between risk factors for falling and the quality of life in older adults. BMC Public Health 2005; 5:90.

28. Chaimowicz F, Ferreira TJXM, Miguel DFA. Use of psychoactive drugs and related falls among older people living in a community in Brazil. Rev Saúde Pública 2000; 34:631-5.

29. Coutinho ES, Fletcher A, Bloch KV, Rodrigues LC. Risk factors for falls with severe fracture in elderly people living in a middle-income country: a case control study. BMC Geriatr 2008; 8:21.

30. Halil M, Ulger Z, Cankurtaran M, Shorbagi A, Yavuz BB, Dede D, et al. Falls and the elderly: is there any difference in the developing world? A crosssectional study from Turkey. Arch Gerontol Geriatr 2006; 43:351-9.

31. Fabrício SCC, Rodrigues RAP, Costa Junior ML. Causas e conseqüências de quedas de idosos atendidos em hospital público. Rev Saúde Pública 2004; 38:93-9.

32. Aschkenasy MT, Rothenhaus TC. Trauma and falls in the elderly. Emerg Med Clin North Am 2006; 24:413-32.

33. Reyes-Ortiz CA, Al Snih S, Markides KS. Falls among elderly persons in Latin America and the Caribbean and among elderly Mexican-Americans. Rev Panam Salud Pública 2005; 17:362-9.

34. Oliver D, Connelly JB, Victor CR, Shaw FE, Whitehead A, Genc Y, et al. Strategies to prevent falls and fractures in hospitals and care homes and effect of cognitive impairment: systematic review and meta-analyses. BMJ 2007; 334:82.

35. Stevens JA, Corso PS, Finkelstein EA, Miller TR. The costs of fatal and non-fatal falls among older adults. Inj Prev 2006; 12:290-5.

36. Bertolucci PH, Brucki SM, Campacci SR, Juliano Y. The Mini-Mental State Examination in a general population: impact of educational status. Arq Neuropsiquiatr 1994; 52:1-7.

37. Mustard CA, Mayer T. Case-control study of exposure to medication and the risk of injurious falls requiring hospitalization among nursing home residents. Am J Epidemiol 1997; 145:738-45.

38. Ray WA, Thapa PB, Gideon P. Benzodiazepines and the risk of falls in nursing home residents. J Am Geriatr Soc 2000; 48:682-5.

39. Coutinho ES, Bloch KV, Rodrigues LC. Characteristics and circumstances of falls leading to severe fractures in elderly people in Rio de Janeiro, Brazil. Cad Saúde Pública 2009; 25:455-9.

40. Lebrão ML, Laurenti R. Saúde, bem-estar e envelhecimento: o estudo SABE no município de São Paulo. Rev Bras Epidemiol 2005; 8:127-41.

41. Daal JO, van Lieshout JJ. Falls and medications in the elderly. Neth J Med 2005; 63:91-6.

42. Siqueira AB, Cordeiro RC, Perracini MR, Ramos LR. Impacto funcional da internação hospitalar de pacientes idosos. Rev Saúde Pública 2004; 38:687-94.

43. Perracini MR, Ramos LR. Fatores associados a quedas em uma coorte de idosos residentes na comunidade. Rev Saúde Pública 2002; 36:709-16.

44. Chaimowicz F, Greco DB. Dinâmica da institucionalização de idosos em Belo Horizonte, Brasil. Rev Saúde Pública 1999; 33:454-60. 
45. Matsudo SM, Matsudo VKR, Barros Neto TL. Impacto do envelhecimento nas variáveis antropométricas, neuromotoras e metabólicas da aptidão física. Rev Bras Ciênc Mov 2000; 8:21-32.

46. Litvak J. El envejecimiento de la población: un desafío que vá más allá del ano 2000. Bol Oficina Sanit Panam 1990; 109:1-5.

47. Fried LP, Tangen CM, Walston J, Newman AB, Hirsch C, Gottdiener J, et al. Frailty in older adults: evidence for a phenotype. J Gerontol A Biol Sci Med Sci 2001; 56:M146-56.
48. Myers AH, Young Y, Langlois JA. Prevention of falls in the elderly. Bone 1996; 18:87-101.

49. Lima-Costa MF, Barreto SM, Giatti L. Condições de saúde, capacidade funcional, uso de serviços de saúde e gastos com medicamentos da população idosa brasileira: um estudo descritivo baseado na Pesquisa Nacional por Amostra de Domicílios. Cad Saúde Pública 2003; 19:735-43.

Recebido em 28/Out/2008

Versão final reapresentada em 15/Ago/2009 Aprovado em 08/Set/2009 